\title{
Symmetry Analysis and Conservation Laws of the Boundary Value Problems for Time-Fractional Generalized Burgers' Differential Equation
}

\author{
Gulistan Iskenderoglu ${ }^{1 *}$ and Dogan Kaya ${ }^{1}$ \\ ${ }^{1}$ Istanbul Commerce University, Faculty of Art and Science, Istanbul, Turkey \\ *Corresponding author E-mail: gulistan.iskandarova@istanbulticaret.edu.tr
}

\section{Article Info}

Keywords: Boundary value problem, Conservation laws, Generalized Burgers' equation, Lie groups method, Riemann-Liouville derivative 2010 AMS: 35G30, 35L65, 4H15, $26 A 33$

Received: 29 July 2019

Accepted: 10 December 2019

Available online: 20 December 2019

\begin{abstract}
Many physical phenomena in nature can be described or modeled via a differential equation or a system of differential equations. In this work, we restrict our attention to research a solution of fractional nonlinear generalized Burgers' differential equations. Thereby we find some exact solutions for the nonlinear generalized Burgers' differential equation with a fractional derivative, which has domain as $\mathbb{R}^{2} \times \mathbb{R}^{+}$. Here we use the Lie groups method. After applying the Lie groups to the boundary value problem we get the partial differential equations on the domain $\mathbb{R}^{2}$ with reduced boundary and initial conditions. Also, we find conservation laws for the nonlinear generalized Burgers' differential equation.
\end{abstract}

\section{Introduction}

The research of exact solutions plays an important role in the study of nonlinear systems. Many methods as the inverse scattering method [1], Hirota bilinear method [2], Lie symmetry analysis [3,4], CK (Clarkson-Kruskal) method [5,6], etc. have been developed to find these exact physically significant solutions of the partial differential equation, although this is rather difficult. Our work in this area is to use Lie transformation methods and its analysis to search exact solutions to fractional nonlinear partial differential equations. It is known that the Lie group method is a powerful and direct approach to the construction of exact solutions of nonlinear differential equations. Essentially, the symmetry analysis is aimed at using the symmetry of the equation. The process thus obtained reduces the complexity of the given equation. Even though physical phenomena are mostly based on searching the solution of the underlying nonlinear model equations, it is too difficult to find a general solution of the fractional nonlinear partial differential equation. There is no existing general theory for nonlinear partial differential equations. While there is no existing general theory for nonlinear partial differential equations, many special cases have yielded to appropriate changes of variable [7-11]. In fact, transformations are perhaps the most powerful tool currently available in this area [12-14]. Ivanova, Sophocleous and Tracin in [15] investigated the Lie symmetry analysis of $(2+1)$ - dimensional variable coefficient Burgers differential equation of the form

$$
u_{t}=A(t) u_{x x}+B(t) u_{y y}+u u_{x} .
$$

They obtained the symmetries, according them conservation laws and some analytical solutions for above equation. Later Abd-el-Malek and Amin in [16] studied the symmetry analysis of the generalized (1+1)-dimensional Burgers differential equation in the form

$$
u_{t}+\alpha\left(u^{n}\right)_{x}=\beta g(t)\left(u^{n}\right)_{x x},
$$

with boundary and initial conditions $u(0, x) \longrightarrow \infty$, for $x>0, u(t, 0)=\gamma r(t)$, for $t>0, \gamma \neq 0$, and $\lim _{x \rightarrow \infty} u(t, x) \longrightarrow \infty$, for $t>0$.

Some recent studies of Burgers differential equation the reader can see in $[17,18]$.

In this research, we show the applying of Lie group analysis to study (2+1)-dimensional time-fractional generalized Burgers' differential equation with boundary and initially conditions: 


$$
\begin{array}{cc}
\frac{\partial^{\alpha} u}{\partial t^{\alpha}}+P \nabla\left(u^{n}\right)=R g(t) \triangle\left(u^{n}\right), \\
u(0, x, y) \longrightarrow \infty, & (x, y) \in \mathbb{R}^{2} \\
u(t, 0,0)=\Phi(t), & t \in[0, \infty), \\
\lim _{(x, y) \rightarrow(\infty, \infty)} u(t, x, y) \longrightarrow \infty, & t \in[0, \infty) .
\end{array}
$$

$\operatorname{Here}(x, y) \in \mathbb{R}^{2}, t \in \mathbb{R}^{+}, 0<\alpha \leq 1, n>1, P, R \neq 0$ and $\frac{\partial^{\alpha} u}{\partial t^{\alpha}}$ is a fractional derivative which is considered in the Riemann-Liouville terms as [19]

$$
\frac{\partial^{\alpha} f(t)}{\partial t^{\alpha}}= \begin{cases}\frac{1}{\Gamma(n-\alpha)} \frac{d^{n}}{d t^{n}} \int_{0}^{t} \frac{f(\tau)}{(t-\tau)^{\alpha+1-n}} d \tau, & \text { if } \quad \alpha \notin \mathbb{N}, \quad n-1<\alpha<n, \quad n \in \mathbb{N}, \\ \frac{d^{n}}{d t^{n}} f(t), & \text { if } \quad \alpha, n \in \mathbb{N} .\end{cases}
$$

Moreover we investigate the conservation laws for above equation by using Ibragimov's theorem for fractional derivative equations [7,20]. The Lie group or Lie symmetry analysis allows us to see that the underlying symmetry algebra of the equation reduce the dimension, it is since each of the time-fractional equations is invariant under time translation symmetry. So, by using the Lie symmetry, we show that the fractional partial differential equation with the domain $\mathbb{R}^{2} \times \mathbb{R}^{+}$can be transformed into a nonlinear fractional partial differential equation with the domain $\mathbb{R}^{2}$.

\section{Symmetry analysis for time fractional partial differential equation}

Consider a time-fractional partial differential equations with three independent variables $x>0, y>0$, and $t>0$ as following:

$$
F\left(x, y, t, u, \partial_{t}^{\alpha} u, u_{x}, u_{y}, u_{x x}, u_{y y}\right)=0, \quad 0<\alpha \leq 1,
$$

where $\partial_{t}^{\alpha} u$ is Riemann-Liouville fractional derivative of $u$.

A one parameter Lie symmetry transformations acting on a space of three independent variables $(t, x, y)$ and depended variable $u$ are determined as

$$
\begin{aligned}
& \bar{t}=t+\varepsilon \tau(t, x, y, u)+O\left(\varepsilon^{2}\right), \\
& \bar{x}=x+\varepsilon \xi_{1}(t, x, y, u)+O\left(\varepsilon^{2}\right), \\
& \bar{y}=y+\varepsilon \xi_{2}(t, x, y, u)+O\left(\varepsilon^{2}\right), \\
& \bar{u}=u+\varepsilon \eta(t, x, y, u)+O\left(\varepsilon^{2}\right),
\end{aligned}
$$

where $\varepsilon>0$ is an infinitesimal group parameter. The infinitesimal generator associated with the above transformations can be written as:

$$
X=\xi_{1}(t, x, y, u) \frac{\partial}{\partial x}+\xi_{2}(t, x, y, u) \frac{\partial}{\partial y}+\tau(t, x, y, u) \frac{\partial}{\partial t}+\eta(t, x, y, u) \frac{\partial}{\partial u}
$$

with $\xi_{1}=\left.\frac{d \bar{x}}{d \varepsilon}\right|_{\varepsilon=0}, \xi_{2}=\left.\frac{d \bar{y}}{d \varepsilon}\right|_{\varepsilon=0}, \tau=\left.\frac{d \bar{t}}{d \varepsilon}\right|_{\varepsilon=0}$ and $\eta=\left.\frac{d \bar{u}}{d \varepsilon}\right|_{\varepsilon=0}$. According to the infinitesimal invariant criterion (2.2), prolongation $p r^{(\alpha, 2)} X$ to equation (2.1) has the form

$$
\left.p r^{(\alpha, 2)} X(E)\right|_{E=0}=0, \quad E=F\left(t, x, y, u, \partial_{t}^{\alpha} u, u_{x}, u_{x x}\right)=0,
$$

here the operator $p r^{(\alpha, 2)} X$ takes the following form

$$
p r^{(\alpha, 2)} X=X+\eta_{\alpha}^{t} \partial_{t}^{\alpha} u+\eta_{1}^{x} \partial_{u_{x}}+\eta_{2}^{x} \partial_{u_{x x}}+\eta_{1}^{y} \partial_{u_{y}}+\eta_{2}^{y} \partial_{u_{y y}},
$$

where

$$
\begin{gathered}
\eta_{\alpha}^{t}=D_{t}^{\alpha}(\eta)+\xi_{1} D_{t}^{\alpha}\left(u_{x}\right)-D_{t}^{\alpha}\left(\xi_{1} u_{x}\right)+\xi_{2} D_{t}^{\alpha}\left(u_{y}\right)-D_{t}^{\alpha}\left(\xi_{2} u_{y}\right)+D_{t}^{\alpha}\left(u D_{t}(\tau)\right)-D_{t}^{\alpha+1}(\tau u)+\tau D_{t}^{\alpha+1}(u), \\
\eta_{1}^{x}=D_{x} \eta-u_{x} D_{x} \xi_{1}-u_{y} D_{x} \xi_{2}-u_{t} D_{x} \tau, \\
\eta_{2}^{x}=D_{x} \eta_{1}^{x}-u_{x x} D_{x} \xi_{1}-u_{x y} D_{x} \xi_{2}-u_{x t} D_{x} \tau, \\
\eta_{1}^{y}=D_{y} \eta-u_{x} D_{y} \xi_{1}-u_{y} D_{y} \xi_{2}-u_{t} D_{y} \tau, \\
\eta_{2}^{y}=D_{y} \eta_{1}^{y}-u_{y x} D_{y} \xi_{1}-u_{y y} D_{y} \xi_{2}-u_{y t} D_{y} \tau,
\end{gathered}
$$

with $D_{i}$ is the total derivative

$$
D_{i}=\partial_{i}+u_{i} \partial_{u}+u_{i t} \partial_{u_{t}}+u_{j t} \partial_{u_{t}}+u_{i i} \partial_{u_{i}}+u_{j j} \partial_{u_{j}}+\ldots
$$

and $D_{t}^{\alpha}$ is a fractional derivative operator with respect to $t$. 
The expression for $\eta_{1}^{x}, \eta_{1}^{y}, \eta_{2}^{x}$, and $\eta_{2}^{y}$ in (2.3) can be easily obtained [4,21], here we concentrate our attention on $\eta_{\alpha}^{t}$. Using the generalized Leibnitz rule, that was given in [22]

$$
D_{t}^{\alpha}(f(t) g(t))=\sum_{n=0}^{\infty}\left(\begin{array}{l}
\alpha \\
n
\end{array}\right) D_{t}^{\alpha-n} f(t) D_{t}^{n} g(t), \quad\left(\begin{array}{l}
\alpha \\
n
\end{array}\right)=\frac{(-1)^{n-1} \alpha \Gamma(n-\alpha)}{\Gamma(1-\alpha) \Gamma(n+1)}
$$

So we get

$$
\begin{aligned}
& \xi_{1} D_{t}^{\alpha}\left(u_{x}\right)-D_{t}^{\alpha}\left(\xi_{1} u_{x}\right)=-\sum_{n=1}^{\infty}\left(\begin{array}{l}
\alpha \\
n
\end{array}\right) D_{t}^{\alpha-n}\left(u_{x}\right) D_{t}^{n}\left(\xi_{1}\right), \\
& \xi_{2} D_{t}^{\alpha}\left(u_{y}\right)-D_{t}^{\alpha}\left(\xi_{2} u_{y}\right)=-\sum_{n=1}^{\infty}\left(\begin{array}{l}
\alpha \\
n
\end{array}\right) D_{t}^{\alpha-n}\left(u_{y}\right) D_{t}^{n}\left(\xi_{2}\right),
\end{aligned}
$$

and

$$
D_{t}^{\alpha}\left(u D_{t}(\tau)\right)-D_{t}^{\alpha+1}(\tau u)+\tau D_{t}^{\alpha+1}(u)=-\alpha D_{t}(\tau) D_{t}^{\alpha}(u)-\sum_{n=1}^{\infty}\left(\begin{array}{c}
\alpha \\
n+1
\end{array}\right) D_{t}^{\alpha-n}(u) D_{t}^{n+1}(\tau) .
$$

Thereby we get the expression

$$
\eta_{\alpha}^{t}=D_{t}^{\alpha}(\eta)-\sum_{n=1}^{\infty}\left(\begin{array}{c}
\alpha \\
n
\end{array}\right) D_{t}^{\alpha-n}\left(u_{x}\right) D_{t}^{n}\left(\xi_{1}\right)-\sum_{n=1}^{\infty}\left(\begin{array}{c}
\alpha \\
n
\end{array}\right) D_{t}^{\alpha-n}\left(u_{y}\right) D_{t}^{n}\left(\xi_{2}\right)-\alpha D_{t}(\tau) D_{t}^{\alpha}(u)-\sum_{n=1}^{\infty}\left(\begin{array}{c}
\alpha \\
n+1
\end{array}\right) D_{t}^{\alpha-n}(u) D_{t}^{n+1}(\tau) .
$$

According to the compound function of the chain rule [23] we get

$$
\frac{d^{m} f(g(t))}{d t^{m}}=\sum_{k=0}^{m} \sum_{r=0}^{k}\left(\begin{array}{l}
k \\
r
\end{array}\right) \frac{1}{k !}(-g(t))^{r} \frac{d^{m}}{d t^{m}}\left(g(t)^{k-r}\right) \frac{d^{k} f(g)}{d g^{k}}
$$

Thus infinitesimal $\eta_{\alpha}^{t}$ takes a form

$$
\begin{gathered}
\eta_{\alpha}^{t}=\frac{\partial^{\alpha} \eta}{\partial t^{\alpha}}+\left(\eta_{u}-\alpha\left(\tau_{t}+u_{t} \tau_{u}\right)\right) \frac{\partial^{\alpha} u}{\partial t^{\alpha}}-u \frac{\partial^{\alpha} \eta_{u}}{\partial t^{\alpha}}+\mu \\
+\sum_{n=1}^{\infty}\left[\left(\begin{array}{c}
\alpha \\
n
\end{array}\right) \frac{\partial^{n} \eta_{u}}{\partial t^{n}}-\left(\begin{array}{c}
\alpha \\
n+1
\end{array}\right) D_{t}^{n+1} \tau\right] D_{t}^{\alpha-n} u-\sum_{n=1}^{\infty}\left(\begin{array}{c}
\alpha \\
n
\end{array}\right) D_{t}^{n}\left(\xi_{1}\right) D_{t}^{\alpha-n}\left(u_{x}\right)-\sum_{n=1}^{\infty}\left(\begin{array}{c}
\alpha \\
n
\end{array}\right) D_{t}^{\alpha-n}\left(u_{y}\right) D_{t}^{n}\left(\xi_{2}\right),
\end{gathered}
$$

where

$$
\mu=\sum_{n=2}^{\infty} \sum_{m=2}^{n} \sum_{k=2}^{m} \sum_{r=0}^{k}\left(\begin{array}{l}
\alpha \\
n
\end{array}\right)\left(\begin{array}{l}
n \\
m
\end{array}\right)\left(\begin{array}{l}
k \\
r
\end{array}\right) \frac{1}{k !} \frac{t^{n-\alpha}(-u)^{r}}{\Gamma(n+1-\alpha)} \frac{\partial^{m}}{\partial t^{m}}\left(u^{k-r}\right) \frac{\partial^{n-m+k} \eta}{\partial t^{n-m} \partial u^{k}}
$$

\section{Symmetry analysis for time-fractional nonlinear generalized Burgers' differential equation}

After some easy mathematical transformations our equation (1.1) can be written in the form

$$
\frac{\partial^{\alpha} u}{\partial t^{\alpha}}+n P u^{n-1}\left(u_{x}+u_{y}\right)=n R g(t)\left((n-1) u^{n-2}\left(u_{x}^{2}+u_{y}^{2}\right)+u^{n-1}\left(u_{x x}+u_{y y}\right)\right) .
$$

By substitution of transformations (2.2) and (2.3) into (3.1) and equating the multiplier of $\varepsilon$ to zero we get that, for the fractional nonlinear generalized Burgers' differential equation (3.1) the invariance criterion takes the form

$$
\begin{gathered}
\eta_{\alpha}^{t}+n(n-1) P u^{n-2} \eta\left(u_{x}+u_{y}\right)+n P u^{n-1}\left(\eta_{1}^{x}+\eta_{1}^{y}\right)-n(n-1)(n-2) \operatorname{Rg}(t) u^{n-3} \eta\left(u_{x}^{2}+u_{y}^{2}\right) \\
-2 n(n-1) \operatorname{Rg}(t) u^{n-2}\left(\eta_{1}^{x}+\eta_{1}^{y}\right)-n(n-1) \operatorname{Rg}^{\prime}(t) u^{n-2}\left(u_{x}^{2}+u_{y}^{2}\right) \tau \\
-n(n-1) \operatorname{Rg}(t) u^{n-2}\left(u_{x x}+u_{y y}\right) \eta-n \operatorname{Rg}^{\prime}(t) u^{n-1}\left(u_{x x}+u_{y y}\right) \tau-n \operatorname{Rg}(t) u^{n-1}\left(\eta_{2}^{x}+\eta_{2}^{y}\right)=0 .
\end{gathered}
$$

Substituting the extended infinitesimals (2.3) into the equation (3.2) we get following system of differential equations:

$$
\partial_{u} \xi_{1}=\partial_{u} \xi_{2}=\partial_{t} \xi_{1}=\partial_{t} \xi_{2}=\partial_{u} \tau=\partial_{x} \tau=\partial_{y} \tau=\eta_{u u}=0
$$

$P \eta\left(n^{2}-n\right)+R g(t) \partial_{x} \eta\left(2 n-2 n^{2}\right)+u\left(n P \alpha \partial_{t} \tau-n P\left(\partial_{y} \xi_{1}+\partial_{x} \xi_{1}\right)+n R g(t)\left(\partial_{y y} \xi_{1}+\partial_{x x} \xi_{1}\right)+2 n R g(t) \partial_{x u} \eta\right)=0$,

$P \eta\left(n^{2}-n\right)+R g(t) \partial_{y} \eta\left(2 n-2 n^{2}\right)+u\left(n P \alpha \partial_{t} \tau-n P\left(\partial_{y} \xi_{2}+\partial_{x} \xi_{2}\right)+n R g(t)\left(\partial_{y y} \xi_{2}+\partial_{x x} \xi_{2}\right)+2 n R g(t) \partial_{y u} \eta\right)=0$,

$$
\begin{gathered}
R g(t) \eta\left(n-n^{2}\right)+u\left(-n R^{\prime}(t) \tau-n R \alpha g(t) \partial_{t} \tau+2 n R g(t) \partial_{x} \xi_{1}\right)=0 \\
R g(t) \eta\left(n-n^{2}\right)+u\left(-n R^{\prime}(t) \tau-n R \alpha g(t) \partial_{t} \tau+2 n R g(t) \partial_{x} \xi_{2}\right)=0 \\
\partial_{u t} \eta-\frac{\alpha-1}{2} \partial_{t t} \tau=0, \\
\frac{\partial^{\alpha} \eta}{\partial t^{\alpha}}-u \frac{\partial^{\alpha} \eta_{u}}{\partial t^{\alpha}}+n P u^{n-1}\left(\partial_{x} \eta+\partial_{y} \eta\right)-n R g(t) u^{n-1}\left(\partial_{x x} \eta+\partial_{y y} \eta\right)=0
\end{gathered}
$$


In below we study some cases and obtain generating infinitesimal operators for classification of solutions of the equation.

Case 1: For arbitrary $g(t)$ and $0<\alpha \leq 1$ we get infinitesimals as

$$
\begin{aligned}
\xi_{1} & =c_{1}, \\
\xi_{2} & =c_{2}, \\
\tau & =0, \\
\eta & =0,
\end{aligned}
$$

here $c_{1}$ and $c_{2}$ are arbitrary constants and there are two infinitesimal operators

$$
X_{1}=\frac{\partial}{\partial x} \quad X_{2}=\frac{\partial}{\partial y}
$$

Case 2: For $g(t)=1$ we get following infinitesimals

$$
\begin{gathered}
\xi_{1}=c_{1}, \\
\xi_{2}=c_{2}, \\
\tau=c_{3} t+c_{4} \\
\eta=-\frac{\alpha c_{3}}{n-1} u
\end{gathered}
$$

here $c_{1}, c_{2}, c_{3}$, and $c_{4}$ are arbitrary constants and thus we obtain two additional infinitesimal operators

$$
X_{3}=\frac{\partial}{\partial t}, \quad X_{4}=t \frac{\partial}{\partial t}+\frac{\alpha}{1-n} u \frac{\partial}{\partial u} .
$$

Case 3: For $g(t)=t^{b}$ with $b \neq 0$ we have infinitesimals as

$$
\begin{gathered}
\xi_{1}=c_{5} x+c_{6} \\
\xi_{2}=c_{5} x+c_{7} \\
\tau=c_{5} \frac{t}{b} \\
\eta=\frac{b-\alpha}{b(n-1)} c_{5} u
\end{gathered}
$$

here $c_{5}, c_{6}$, and $c_{7}$ are arbitrary constants and there is one additional infinitesimal operator

$$
X_{3}=x \frac{\partial}{\partial x}+y \frac{\partial}{\partial y}+\frac{t}{b} \frac{\partial}{\partial t}+\frac{b-\alpha}{b(n-1)} u \frac{\partial}{\partial u} .
$$

Case 4: For $g(t)=e^{t}$ we obtain following infinitesimals in a form

$$
\begin{gathered}
\xi_{1}=c_{6} x+c_{7}, \\
\xi_{2}=c_{6} y+c_{8}, \\
\tau=c_{6}, \\
\eta=\frac{c_{6}}{n-1} u
\end{gathered}
$$

here $c_{6}, c_{7}$ and $c_{8}$ are arbitrary constants and we have one additional infinitesimal operator

$$
X_{3}=x \frac{\partial}{\partial x}+y \frac{\partial}{\partial y}+\frac{\partial}{\partial t}+\frac{1}{n-1} u \frac{\partial}{\partial u} .
$$

\section{Symmetry reductions of the time fractional nonlinear generalized Burgers' differential equation}

Now, we obtain similarity reductions and present the reduced nonlinear fractional ordinary differential equations. Also we classify the corresponding group invariant solutions of the fractional nonlinear generalized Burgers' equation.

Case 2: For $g(t)=1$ we have four infinitesimal operators

$$
X_{1}=\frac{\partial}{\partial x}, \quad X_{2}=\frac{\partial}{\partial y}, \quad X_{3}=\frac{\partial}{\partial t}, \quad X_{4}=t \frac{\partial}{\partial t}+\frac{\alpha}{1-n} u \frac{\partial}{\partial u} .
$$

The similarity variables for infinitesimal operator $X_{1}$ and $X_{2}$ can be found by solving the corresponding characteristic equation

$$
\frac{d x}{1}=\frac{d y}{0}=\frac{d t}{0}=\frac{d u}{0} \quad \frac{d x}{0}=\frac{d y}{1}=\frac{d t}{0}=\frac{d u}{0} .
$$

Thus we obtain the similarity reduction $u=\phi(t)$, by substituting which into (1.1) we get

$$
D_{t}^{\alpha} \phi(t)=0 \text {. }
$$

Thereby the exact solution of time fractional nonlinear generalized Burgers' differential equation (1.1) with $X_{1}$ and $X_{2}$ is

$$
u(t, x, y)=c t^{\alpha-1},
$$

where $c$ is arbitrary constant.

For infinitesimal operator $X_{3}$ the corresponding characteristic equation is in a form

$$
\frac{d x}{0}=\frac{d y}{0}=\frac{d t}{1}=\frac{d u}{0} .
$$


This equation gives us a similarity reduction $u=\phi(x, y)$, by substituting which into (1.1) we have

$$
u(t, x, y)=0 .
$$

And the similarity variables for infinitesimal operator $X_{4}$ can be found by solving the corresponding characteristic equation

$$
\frac{d x}{0}=\frac{d y}{0}=\frac{d t}{t}=\frac{d u}{\frac{\alpha u}{1-n}} .
$$

Here we have $u=t^{\frac{\alpha}{1-n}} \phi(x, y)$ similarity reduction, by substituting it into (1.1) we get

$$
\begin{gathered}
\frac{1}{\Gamma(1-\alpha)} \frac{d}{d t} \int_{0}^{t}(t-s)^{-\alpha} s^{\frac{\alpha}{1-n}} \phi(x, y) d s+n P t^{\frac{\alpha_{n}}{1-n}}(\phi(x, y))^{n-1}\left(\partial_{x} \phi(x, y)+\partial_{y} \phi(x, y)\right) \\
\left.-R t^{\frac{\alpha_{n}}{1-n}}\left(n(\phi(x, y))^{n-1}\left(\partial_{x x} \phi(x, y)+\partial_{y y} \phi(x, y)\right)+n(n-1)(\phi(x, y))^{n-2}\left(\left(\partial_{x} \phi(x, y)\right)^{2}+\partial_{y} \phi(x, y)\right)^{2}\right)\right)=0 .
\end{gathered}
$$

After some easy transformations we obtain following nonlinear ordinary differential equation

$$
\begin{gathered}
\frac{\Gamma\left(1+\frac{\alpha}{(1-n)}\right)}{\Gamma\left(1-\alpha+\frac{\alpha}{(1-n)}\right)} \phi(x, y)+ \\
n P(\phi(x, y))^{n-1}\left(\partial_{x} \phi(x, y)+\partial_{y} \phi(x, y)\right)-R n(\phi(x, y))^{n-1}\left(\partial_{x x} \phi(x, y)+\partial_{y y} \phi(x, y)\right) \\
\left.-R n(n-1)(\phi(x, y))^{n-2}\left(\left(\partial_{x} \phi(x, y)\right)^{2}+\partial_{y} \phi(x, y)\right)^{2}\right)=0 .
\end{gathered}
$$

Case 3: For $g(t)=t^{b}$ with $b \neq 0$ we have three infinitesimal operators

$$
X_{1}=\frac{\partial}{\partial x}, \quad X_{2}=\frac{\partial}{\partial y}, \quad X_{3}=x \frac{\partial}{\partial x}+y \frac{\partial}{\partial y}+\frac{t}{b} \frac{\partial}{\partial t}+\frac{b-\alpha}{b(n-1)} u \frac{\partial}{\partial u} .
$$

The third infinitesimal operator by solving the corresponding characteristic equations

$$
\frac{d x}{x}=\frac{d y}{y}=\frac{b d t}{t}=\frac{b d u}{\frac{b-\alpha}{n-1} u}
$$

gives us the similarity reduction

$$
u(x, y, t)=t^{\frac{b-\alpha}{n-1}} \omega\left(p_{1}, p_{2}\right)
$$

where $p_{1}=x t^{-b}$ and $p_{2}=y t^{-b}$.

Case 4: And lastly for $g(t)=e^{t}$ we have three infinitesimal operators

$$
X_{1}=\frac{\partial}{\partial x}, \quad X_{2}=\frac{\partial}{\partial y}, \quad X_{3}=x \frac{\partial}{\partial x}+y \frac{\partial}{\partial y}+\frac{\partial}{\partial t}+\frac{1}{(n-1)} u \frac{\partial}{\partial u} .
$$

The third infinitesimal operator gives us the corresponding characteristic equations

$$
\frac{d x}{x}=\frac{d y}{y}=\frac{d t}{1}=\frac{d u}{\frac{1}{n-1} u}
$$

and a similarity reduction

here $q_{1}=x e^{-t}$, and $q_{2}=y e^{-t}$.

$$
u(x, y, t)=e^{\frac{1}{n-1} t} v\left(q_{1}, q_{2}\right)
$$

\section{Conservation laws}

In this section we will construct the conservation laws of time-fractional nonlinear generalized Burgers' differential equation (2.1) by using Ibragimov's theorem $[24,25]$. Ibragimov proved this theorem for differential equations with integer order. And it was applied to fractional differential equations $[26,27]$.

We will search a vector field $C=\left(C^{t}, C^{x}, C^{x}\right)$, where $C^{t}=C^{t}\left(t, x, y, u, u_{x}, u_{y}, \ldots\right), C^{x}=C^{x}\left(t, x, y, u, u_{x}, u_{y}, \ldots\right)$, and $C^{y}=C^{y}\left(t, x, y, u, u_{x}, u_{y}, \ldots\right)$ is conserved vector for (3.1) on all its solution if it satisfies the following conservation equation $D_{t}\left(C^{t}\right)+D_{x}\left(C^{x}\right)+D_{y}\left(C^{y}\right)=0$, where $C^{t}$, $C^{x}$, and $C^{y}$ are conservation laws for equation (2.1). A formal Lagrangian function for (2.1) is given by

$$
L=v(t, x, y) E .
$$

Here $v(t, x, y)$ is a new dependent variable and

$$
E=\frac{\partial^{\alpha} u}{\partial t^{\alpha}}+n P u^{n-1}\left(u_{x}+u_{y}\right)-n(n-1) R g(t) u^{n-2}\left(u_{x}^{2}+u_{y}^{2}\right)+n R g(t) u^{n-1}\left(u_{x x}+u_{y y}\right) .
$$

The Euler-Lagrange operator with respect to $u$ is defined by [27,28]

$$
\frac{\delta}{\delta u}=\frac{\partial}{\partial u}+\left(D_{t}^{\alpha}\right)^{*} \frac{\partial}{\partial D_{t}^{\alpha} u}-D_{x} \frac{\partial}{\partial u_{x}}-D_{y} \frac{\partial}{\partial u_{y}}+D_{x}^{2} \frac{\partial}{\partial u_{x x}}+D_{y}^{2} \frac{\partial}{\partial u_{y y}}-\ldots,
$$

where $\left(D_{t}^{\alpha}\right)^{*}$ is adjoint operator of $D_{t}^{\alpha}$ that has a form

$$
\left(D_{t}^{\alpha}\right)^{*}=(-1)_{t}^{n} I_{T}^{n-\alpha}\left(D_{t}^{n}\right) .
$$


By using Euler-Lagrange operator we can define an adjoint equation of equation (3.1) as

$$
\frac{\delta L}{\delta u}=0
$$

After calculations, the equation (5.2) takes a form

$$
\frac{\delta L}{\delta u}=\left(D_{t}^{\alpha}\right)^{*} v-n P u^{n-1}\left(v_{x}+v_{y}\right)-n R g(t) u^{n-1}\left(v_{x x}+v_{y y}\right) .
$$

So, we say that generalized Burgers' equation is nonlinearly self-adjoint if the adjoint equation (5.2) is satisfied for all solution $u$ of equation (3.1) upon a substitution $v=\varphi(t, x, y, u)$ and $\varphi(t, x, y, u) \neq 0$. This substitution allows us use formal Lagrangian as usual classical Lagrangian and construct the conservation laws.

Thus, $x$-component conservation laws for the equation (3.1) have the form [28]

$$
C_{i}^{x}=\xi_{1} L+W_{i}\left(\frac{\partial L}{\partial u_{x}}-D_{x} \frac{\partial L}{\partial u_{x x}}\right)+D_{x}\left(W_{i}\right)\left(\frac{\partial L}{\partial u_{x x}}\right),
$$

here $W_{i}=\eta^{i}-\xi_{1}^{i} u_{x}-\xi_{2}^{i} u_{y}-\tau^{i} u_{t} . y$-component conservation laws for the equation (3.1) have the form [28]

$$
C_{i}^{y}=\xi_{2} L+W_{i}\left(\frac{\partial L}{\partial u_{y}}-D_{y} \frac{\partial L}{\partial u_{y y}}\right)+D_{y}\left(W_{i}\right)\left(\frac{\partial L}{\partial u_{y y}}\right) .
$$

And $t$-component conservation laws for the equation (3.1) have the form

$$
C_{i}^{t}=\sum_{k=0}^{m-1}(-1)^{k} D_{t}^{\alpha-1-k}\left(W_{i}\right) D_{t}^{k}\left(\frac{\partial L}{\partial D_{t}^{\alpha} u}\right)-(-1)^{m} J\left(W_{i}, D_{t}^{m} \frac{\partial L}{\partial D_{t}^{\alpha} u}\right),
$$

for $m-1<\alpha<m$ and $J$ is a integral

$$
J(f, g)=\frac{1}{\Gamma(m-\alpha)} \int_{0}^{t} \int_{t}^{T} \frac{f(x, y, s) g(x, y, p)}{(p-s)^{\alpha+1-m}} d p d s .
$$

Thus, by using (5.1) and above formulas we can find $C^{x}, C^{y}$, and $C^{t}$ for our problem.

Case 1: For arbitrary $g(t)$ we have $W_{1}=u_{x}$ and $W_{2}=u_{y}$ that gives us

$$
\begin{gathered}
C_{1}^{t}=v D_{t}^{\alpha-1}\left(u_{x}\right)+J\left(u_{x}, v_{t}\right), \\
C_{2}^{t}=v D_{t}^{\alpha-1}\left(u_{y}\right)+J\left(u_{y}, v_{t}\right), \\
C_{1}^{x}=v\left(\frac{\partial^{\alpha} u}{\partial t^{\alpha}}+n P u^{n-1}\left(u_{x}+u_{y}\right)-n(n-1) R g(t) u^{n-2}\left(u_{x}^{2}+u_{y}^{2}\right)+n R g(t) u^{n-1}\left(u_{x x}+u_{y y}\right)\right)+ \\
n R g(t) u^{n-1} u_{x x} v-3 n(n-1) R g(t) u^{n-2} v\left(u_{x}\right)^{2}+n P u^{n-1} u_{x} v-n R g(t) u^{n-1} u_{x}\left(u_{x} v_{u}+v_{x}\right), \\
C_{2}^{x}=n R g(t) u^{n-1} u_{x y} v-3 n(n-1) R g(t) u^{n-2} v u_{x} u_{y}+n P u^{n-1} u_{y} v-n R g(t) u^{n-1} u_{y}\left(u_{x} v_{u}+v_{x}\right), \\
C_{1}^{y}=n R g(t) u^{n-1} u_{x y} v-3 n(n-1) \operatorname{Rg}(t) u^{n-2} v u_{x} u_{y}+n P u^{n-1} u_{x} v-n R g(t) u^{n-1} u_{x}\left(u_{y} v_{u}+v_{y}\right), \\
C_{2}^{y}=v\left(\frac{\partial^{\alpha} u}{\partial t^{\alpha}}+n P u^{n-1}\left(u_{x}+u_{y}\right)-n(n-1) R g(t) u^{n-2}\left(u_{x}^{2}+u_{y}^{2}\right)+n R g(t) u^{n-1}\left(u_{x x}+u_{y y}\right)\right)+ \\
n R g(t) u^{n-1} u_{y y} v-3 n(n-1) \operatorname{Rg}(t) u^{n-2} v\left(u_{y}\right)^{2}+n P u^{n-1} u_{y} v-n \operatorname{Rg}(t) u^{n-1} u_{y}\left(u_{y} v_{u}+v_{y}\right) .
\end{gathered}
$$

Case 2: For $g(t)=t^{b}$ we get $W_{1}=u_{x}, W_{2}=u_{y}$, and $W_{3}=x u_{x}+y u_{y}+\frac{t}{b} u_{t}+\frac{\alpha-b}{b(1-n)} u$, thus the corresponding conservation laws are like:

$C_{1}^{t}$ and $C_{2}^{t}$ are the same.

$$
\begin{gathered}
C_{3}^{t}=t v\left(\frac{\partial^{\alpha} u}{\partial t^{\alpha}}+n P u^{n-1}\left(u_{x}+u_{y}\right)-n(n-1) R g(t) u^{n-2}\left(u_{x}^{2}+u_{y}^{2}\right)+n R g(t) u^{n-1}\left(u_{x x}+u_{y y}\right)\right)+ \\
v D_{t}^{\alpha-1}\left(x u_{x}+y u_{y}+\frac{t}{b} u_{t}+\frac{\alpha-b}{b(1-n)} u\right)+J\left(x u_{x}+y u_{y}+\frac{t}{b} u_{t}+\frac{\alpha-b}{b(1-n)} u, v_{t}\right), \\
C_{1}^{x}=v\left(\frac{\partial^{\alpha} u}{\partial t^{\alpha}}+n P u^{n-1}\left(u_{x}+u_{y}\right)-n(n-1) R t^{b} u^{n-2}\left(u_{x}^{2}+u_{y}^{2}\right)+n R t^{b} u^{n-1}\left(u_{x x}+u_{y y}\right)\right)+ \\
n R t^{b} u^{n-1} u_{x x} v-3 n(n-1) R t^{b} u^{n-2} v\left(u_{x}\right)^{2}+n P u^{n-1} u_{x} v-n R t^{b} u^{n-1} u_{x}\left(u_{x} v_{u}+v_{x}\right), \\
C_{2}^{x}=n R t^{b} u^{n-1} u_{x y} v-3 n(n-1) R t^{b} u^{n-2} v u_{x} u_{y}+n P u^{n-1} u_{y} v-n R t^{b} u^{n-1} u_{y}\left(u_{x} v_{u}+v_{x}\right), \\
C_{3}^{x}=x v\left(\frac{\partial^{\alpha} u}{\partial t^{\alpha}}+n P u^{n-1}\left(u_{x}+u_{y}\right)-n(n-1) R t^{b} u^{n-2}\left(u_{x}^{2}+u_{y}^{2}\right)+n R t^{b} u^{n-1}\left(u_{x x}+u_{y y}\right)\right)+ \\
n R t^{b} u^{n-1} v\left(u_{x}+\frac{\alpha-b}{b(1-n)} u_{x}+\frac{t}{b} u_{x t}+y u_{x y}+x u_{x x}\right)+\left(\frac{\alpha-b}{b(1-n)} u+\frac{t}{b} u_{t}+y u_{y}+x u_{x}\right) \\
\quad\left(-3 n(n-1) R t^{b} u^{n-2} v u_{x}+n P u^{n-1} v-n R t^{b} u^{n-1}\left(u_{x} v_{u}+v_{x}\right)\right), \\
C_{1}^{y}=n R t^{b} u^{n-1} u_{x y} v-3 n(n-1) R t^{b} u^{n-2} v u_{x} u_{y}+n P u^{n-1} u_{x} v-n R t^{b} u^{n-1} u_{x}\left(u_{y} v_{u}+v_{y}\right), \\
C_{2}^{y}=v\left(\frac{\partial^{\alpha} u}{\partial t^{\alpha}}+n P u^{n-1}\left(u_{x}+u_{y}\right)-n(n-1) R t^{b} u^{n-2}\left(u_{x}^{2}+u_{y}^{2}\right)+n R t^{b} u^{n-1}\left(u_{x x}+u_{y y}\right)\right)+ \\
n R t^{b} u^{n-1} u_{y y} v-3 n(n-1) R t^{b} u^{n-2} v\left(u_{y}\right)^{2}+n P u^{n-1} u_{y} v-n R t^{b} u^{n-1} u_{y}\left(u_{y} v_{u}+v_{y}\right), \\
C_{3}^{y}=y v\left(\frac{\partial^{\alpha} u}{\partial t^{\alpha}}+n P u^{n-1}\left(u_{x}+u_{y}\right)-n(n-1) R t^{b} u^{n-2}\left(u_{x}^{2}+u_{y}^{2}\right)+n R t^{b} u^{n-1}\left(u_{x x}+u_{y y}\right)\right)+ \\
n R t^{b} u^{n-1} v\left(u_{y}+\frac{\alpha-b}{b(1-n)} u_{y}+\frac{t}{b} u_{y t}+y u_{y y}+x u_{x y}\right)+\left(\frac{\alpha-b}{b(1-n)} u+\frac{t}{b} u_{t}+y u_{y}+x u_{x}\right) \\
\left(-3 n(n-1) R t^{b} u^{n-2} v u_{y}+n P u^{n-1} v-n R t^{b} u^{n-1}\left(u_{y} v_{u}+v_{y}\right)\right) .
\end{gathered}
$$

Case 3: For $g(t)=1$ we obtain $W_{1}=u_{x}, W_{2}=u_{y}, W_{3}=u_{t}$, and $W_{4}=t u_{t}+\frac{\alpha}{1-n} u$, thus the corresponding conservation laws are in the following form: 
$C_{1}^{t}$ and $C_{2}^{t}$ is the same.

$$
\begin{aligned}
& C_{3}^{t}=v\left(\frac{\partial^{\alpha} u}{\partial t^{\alpha}}+n P u^{n-1}\left(u_{x}+u_{y}\right)-n(n-1) R g(t) u^{n-2}\left(u_{x}^{2}+u_{y}^{2}\right)+n R g(t) u^{n-1}\left(u_{x x}+u_{y y}\right)\right)+v D_{t}^{\alpha-1}\left(u_{t}\right)+J\left(u_{t}, v_{t}\right), \\
& C_{4}^{t}=t v\left(\frac{\partial^{\alpha} u}{\partial t^{\alpha}}+n P u^{n-1}\left(u_{x}+u_{y}\right)-n(n-1) R g(t) u^{n-2}\left(u_{x}^{2}+u_{y}^{2}\right)+n R g(t) u^{n-1}\left(u_{x x}+u_{y y}\right)\right)+ \\
& v D_{t}^{\alpha-1}\left(t u_{t}+\frac{\alpha}{1-n} u\right)+J\left(t u_{t}+\frac{\alpha}{1-n} u, v_{t}\right), \\
& C_{1}^{x}=v\left(\frac{\partial^{\alpha} u}{\partial t^{\alpha}}+n P u^{n-1}\left(u_{x}+u_{y}\right)-n(n-1) R g(t) u^{n-2}\left(u_{x}^{2}+u_{y}^{2}\right)+n R g(t) u^{n-1}\left(u_{x x}+u_{y y}\right)\right)+ \\
& n R u^{n-1} u_{x x} v-3 n(n-1) R u^{n-2}\left(u_{x}\right)^{2} v+n P u^{n-1} u_{x} v-n R u^{n-1} u_{x}\left(u_{x} v_{u}+v_{x}\right), \\
& \left.C_{2}^{x}=n R u^{n-1} v u_{x y}-3 n(n-1) R u^{n-2} u_{x} u_{y} v+n P u^{n-1} u_{y} v-n R u^{n-1} u_{y}\left(u_{x} v_{u}+v_{x}\right)\right) \\
& \left.C_{3}^{x}=n R u^{n-1} v u_{x t}-3 n(n-1) R u^{n-2} u_{x} u_{t} v+n P u^{n-1} u_{t} v-n R u^{n-1} u_{t}\left(u_{x} v_{u}+v_{x}\right)\right), \\
& C_{4}^{x}=n R u^{n-1} v\left(t u_{x t}+\frac{\alpha}{n-1} u_{x}\right)+\left(t u_{t}+\frac{\alpha}{n-1} u\right)\left(-3 n(n-1) R u^{n-2} u_{x} v+n P u^{n-1} v-n R u^{n-1}\left(u_{x} v_{u}+v_{x}\right)\right), \\
& \left.C_{1}^{y}=n R u^{n-1} v u_{x y}-3 n(n-1) R u^{n-2} u_{x} u_{y} v+n P u^{n-1} u_{x} v-n R u^{n-1} u_{x}\left(u_{y} v_{u}+v_{y}\right)\right), \\
& C_{2}^{y}=v\left(\frac{\partial^{\alpha} u}{\partial t^{\alpha}}+n P u^{n-1}\left(u_{x}+u_{y}\right)-n(n-1) R g(t) u^{n-2}\left(u_{x}^{2}+u_{y}^{2}\right)+n R g(t) u^{n-1}\left(u_{x x}+u_{y y}\right)\right)+ \\
& \left.n R u^{n-1} u_{y y} v-3 n(n-1) R u^{n-2}\left(u_{y}\right)^{2} v+n P u^{n-1} u_{y} v-n R u^{n-1} u_{y}\left(u_{y} v_{u}+v_{y}\right)\right), \\
& \left.\left.C_{3}^{y}=n R u^{n-1} v u_{y t}-3 n(n-1) R u^{n-2} u_{y} u_{t} v+n P u^{n-1} u_{t} v-n R u^{n-1} u_{t}\left(u_{y} v_{u}+v_{y}\right)\right)\right), \\
& C_{4}^{y}=n R u^{n-1} v\left(t u_{y t}+\frac{\alpha}{n-1} u_{y}\right)+\left(t u_{t}+\frac{\alpha}{n-1} u\left(-3 n(n-1) R u^{n-2} u_{y} v+n P u^{n-1} v-n R u^{n-1}\left(u_{y} v_{u}+v_{y}\right)\right)\right) .
\end{aligned}
$$

Case 4: For $g(t)=e^{t}$ we have $W_{1}=u_{x}, W_{2}=u_{y}$, and $W_{2}=x u_{x}+y u_{y}+u_{t}+\frac{1}{1-n} u$, and thus the corresponding conservation laws are:

$C_{1}^{t}$ and $C_{2}^{t}$ is the same.

$$
\begin{aligned}
& C_{3}^{t}=v\left(\frac{\partial^{\alpha} u}{\partial t^{\alpha}}+n P u^{n-1}\left(u_{x}+u_{y}\right)-n(n-1) R e^{t} u^{n-2}\left(u_{x}^{2}+u_{y}^{2}\right)+n \operatorname{Re}^{t} u^{n-1}\left(u_{x x}+u_{y y}\right)\right)+ \\
& v D_{t}^{\alpha-1}\left(x u_{x}+y u_{y}+u_{t}+\frac{1}{1-n} u\right)+J\left(x u_{x}+y u_{y}+u_{t}+\frac{1}{1-n} u, v_{t}\right), \\
& C_{1}^{x}=v\left(\frac{\partial^{\alpha} u}{\partial t^{\alpha}}+n P u^{n-1}\left(u_{x}+u_{y}\right)-n(n-1) \operatorname{Re}^{t} u^{n-2}\left(u_{x}^{2}+u_{y}^{2}\right)+n \operatorname{Re}^{t} u^{n-1}\left(u_{x x}+u_{y y}\right)\right)+ \\
& n R e^{t} u^{n-1} u_{x x} v-3 n(n-1) \operatorname{Re}^{t} u^{n-2}\left(u_{x}\right)^{2} v+n P u^{n-1} u_{x} v-n R e^{t} u^{n-1} u_{x}\left(u_{x} v_{u}+v_{x}\right), \\
& C_{2}^{x}=n R e^{t} u^{n-1} u_{x y} v-3 n(n-1) R e^{t} u^{n-2} u_{x} u_{y} v+n P u^{n-1} u_{y} v-n R e^{t} u^{n-1} u_{y}\left(u_{x} v_{u}+v_{x}\right) \text {, } \\
& C_{3}^{x}=v x\left(\frac{\partial^{\alpha} u}{\partial t^{\alpha}}+n P u^{n-1}\left(u_{x}+u_{y}\right)-n(n-1) R e^{t} u^{n-2}\left(u_{x}^{2}+u_{y}^{2}\right)+n \operatorname{Re}^{t} u^{n-1}\left(u_{x x}+u_{y y}\right)\right)+ \\
& n \operatorname{Re}^{t} u^{n-1}\left(u_{x}+x u_{x x}+y u_{x y}+u_{t x}+\frac{1}{1-n} u_{x}\right)+\left(x u_{x}++y u_{y}+u_{t}+\frac{1}{1-n} u\right) \\
& \left(-3 n(n-1) \operatorname{Re}^{t} u^{n-2} u_{x} v+n P u^{n-1} v-n \operatorname{Re}^{t} u^{n-1}\left(u_{x} v_{u}+v_{x}\right)\right), \\
& C_{1}^{y}=n R e^{t} u^{n-1} u_{y x} v-3 n(n-1) R e^{t} u^{n-2} u_{y} u_{x} v+n P u^{n-1} u_{x} v-n R e^{t} u^{n-1} u_{x}\left(u_{y} v_{u}+v_{y}\right) \text {, } \\
& C_{2}^{y}=v\left(\frac{\partial^{\alpha} u}{\partial t^{\alpha}}+n P u^{n-1}\left(u_{x}+u_{y}\right)-n(n-1) \operatorname{Re}^{t} u^{n-2}\left(u_{x}^{2}+u_{y}^{2}\right)+n \operatorname{Re}^{t} u^{n-1}\left(u_{x x}+u_{y y}\right)\right)+ \\
& n R e^{t} u^{n-1} u_{y y} v-3 n(n-1) R e^{t} u^{n-2}\left(u_{y}\right)^{2} v+n P u^{n-1} u_{y} v-n R e^{t} u^{n-1} u_{y}\left(u_{y} v_{u}+v_{y}\right) \text {, } \\
& C_{3}^{y}=v y\left(\frac{\partial^{\alpha} u}{\partial t^{\alpha}}+n P u^{n-1}\left(u_{x}+u_{y}\right)-n(n-1) R e^{t} u^{n-2}\left(u_{x}^{2}+u_{y}^{2}\right)+n R e^{t} u^{n-1}\left(u_{x x}+u_{y y}\right)\right)+ \\
& n \operatorname{Re}^{t} u^{n-1}\left(x u_{x y}+y u_{y}+u_{y y}+u_{t y}+\frac{1}{1-n} u_{y}\right)+\left(x u_{x}+y u_{y}+u_{t}+\frac{1}{1-n} u\right) \\
& \left(-3 n(n-1) \operatorname{Re}^{t} u^{n-2} u_{y} v+n P u^{n-1} v-n \operatorname{Re}^{t} u^{n-1}\left(u_{y} v_{u}+v_{y}\right)\right) .
\end{aligned}
$$

\section{Symmetry analysis for boundary value problem}

In this section, we will discuss the symmetry analysis for the boundary value problem. Lie symmetry analysis is one of the most widely-applicable methods of finding exact solutions of differential equations, but it was not widely used for solving boundary value problems. And the reason is the initial and boundary conditions are usually are not invariant under any obtained Lie symmetry method transformations [3,29-31]. So, for partial differential equations, an invariant solution found by applying symmetry transformation solves a given boundary value problem, when the symmetry transformation leaves invariant all boundary conditions and the domain of the boundary value problem.

Now, let the $Q$-condition symmetry

$$
Q=\xi_{1}(x, y, t, u) \frac{\partial}{\partial x}+\xi_{2}(x, y, t, u) \frac{\partial}{\partial y}+\tau(x, y, t, u) \frac{\partial}{\partial t}+\eta(x, y, t, u) \frac{\partial}{\partial u},
$$

with

$$
\left.Q_{k}\left(u_{t}-F\left(x, y, u, \frac{\partial u}{\partial x}, \frac{\partial u}{\partial y}, \ldots, \frac{\partial^{k} u}{\partial x^{k}}, \frac{\partial^{k} u}{\partial y^{k}}\right)\right)\right|_{M}=0
$$

and the manifold $M=\left\{u_{t}-F\left(x, y, u, \frac{\partial u}{\partial x}, \frac{\partial u}{\partial y}, \ldots, \frac{\partial^{k} u}{\partial x^{k}}, \frac{\partial^{k} u}{\partial y^{k}}\right)=0, Q(u)=0\right\}$ is admitted by the boundary value problem defined on a domain $\Omega$ :

$$
\begin{gathered}
u_{t}=F\left(x, y, u, \frac{\partial u}{\partial x}, \frac{\partial u}{\partial y}, \ldots, \frac{\partial^{k} u}{\partial x^{k}}, \frac{\partial^{k} u}{\partial y^{k}}\right), \quad(x, y, t) \in \Omega \subset \mathbb{R}^{2} \times \mathbb{R}^{+}, \\
d_{a}(x, y, t)=0: B_{a}\left(t, x, y, u, \frac{\partial u}{\partial x}, \frac{\partial u}{\partial y}, \ldots, \frac{\partial^{k-1} u}{\partial x^{k-1}}, \frac{\partial^{k-1} u}{\partial y^{k-1}}\right)=0, \quad a=1, \ldots, p .
\end{gathered}
$$




$$
l_{c}(x, y, t)=\infty: L_{c}\left(x, y, t, u, \frac{\partial u}{\partial x}, \frac{\partial u}{\partial y}, \ldots, \frac{\partial^{k-1} u}{\partial x^{k-1}}, \frac{\partial^{k-1} u}{\partial y^{k-1}}\right)=0, \quad c=1, \ldots, p_{\infty} .
$$

Here $B_{a}\left(t, x, y, u, \frac{\partial u}{\partial x}, \frac{\partial u}{\partial y}, \ldots, \frac{\partial^{k-1} u}{\partial x^{k-1}}, \frac{\partial^{k-1} u}{\partial y^{k-1}}\right)$ boundary condition on $d_{a}(x, y, t)$. Suppose that the above boundary value problem has a unique solution.

So, for the manifold $M=\left\{l_{c}(x, y, t)=\infty, L_{c}\left(x, y, t, u, \frac{\partial u}{\partial x}, \frac{\partial u}{\partial}, \ldots, \frac{\partial^{k} u}{\partial x^{k}}, \frac{\partial^{k} u}{\partial y^{k}}\right)=0\right\}$ there exist a smooth bijective transformation that maps the manifold $M$ into

$$
M^{*}=\left\{l_{c}^{*}\left(x^{*}, y^{*}, t^{*}\right)=\infty, L_{c}^{*}\left(x^{*}, y^{*}, t^{*}, u, \frac{\partial u}{\partial x^{*}}, \frac{\partial u}{\partial}, \ldots, \frac{\partial^{k^{*}} u}{\partial\left(x^{*}\right)^{k^{*}}}, \frac{\partial^{k^{*}} u}{\partial\left(y^{*}\right)^{k^{*}}}\right)=0\right\} .
$$

Definition 6.1. The symmetry $Q$ which has the form (6.1) is allowed by the boundary value problem (6.2)-(6.4) if:

- $Q_{(k)}\left(u_{t}-F\left(x, y, u, \frac{\partial u}{\partial x}, \frac{\partial u}{\partial y}, \ldots, \frac{\partial^{k} u}{\partial x^{k}}, \frac{\partial^{k} u}{\partial y^{k}}\right)\right)=0$ for $u_{t}=F\left(x, y, u, \frac{\partial u}{\partial x}, \frac{\partial u}{\partial y}, \ldots, \frac{\partial^{k} u}{\partial x^{k}}, \frac{\partial^{k} u}{\partial y^{k}}\right)$;

- $Q d_{a}(x, y, t)=0$ for $d_{a}(x, y, t)=0, a=1, \ldots, p$;

- $Q_{(k)} B_{a}\left(x, y, t, u, \frac{\partial u}{\partial x}, \frac{\partial u}{\partial y}, \ldots, \frac{\partial^{k} u}{\partial x^{k}}, \frac{\partial^{k} u}{\partial y^{k}}\right)=0$ for $B_{a}\left(t, x, y, u, \frac{\partial u}{\partial x}, \frac{\partial u}{\partial y}, \ldots, \frac{\partial^{k} u}{\partial x^{k}}, \frac{\partial^{k} u}{\partial y^{k}}\right)=0$ on $d_{a}(x, y, t)=0, a=1, . ., p$;

- there exist a smooth bijective transformation that maps the manifold $M$ into $M^{*}$ of the same dimensionality;

- $Q^{*} l_{c}^{*}\left(x^{*}, y^{*}, t^{*}\right)=0$ for $l_{c}^{*}\left(x^{*}, y^{*}, t^{*}\right)=0, c=1, \ldots, p_{\infty}$;

- $Q_{\left(k^{*}\right)}^{*} L_{c}\left(x, y, t, u, \frac{\partial u}{\partial x}, \frac{\partial u}{\partial y}, \ldots, \frac{\partial^{k} u}{\partial x^{k}}, \frac{\partial^{k} u}{\partial y^{k}}\right)=0$ for $l_{c}(x, y, t)=\infty, c=1, \ldots, r$.

Let us consider our fractional partial differential equation (1.1) with $\alpha=1$, which defined on the domain $0 \leq t<\infty, x>0$, and $y>0$ with initial and boundary conditions

$$
\begin{gathered}
u_{t}+P n u^{n-1}\left(u_{x}+u_{y}\right)=n R g(t) \\
\left(u^{n-1}\left(u_{x x}+u_{y y}+(n-1) u^{n-2}\left(\left(u_{x}\right)^{2}+\left(u_{y}\right)^{2}\right)\right), \quad n>1, \quad P, R \neq 0\right. \\
u(x, y, 0) \longrightarrow \infty, \quad(x, y) \in \mathbb{R}^{+} \times \mathbb{R}^{+}, \\
u(0,0, t)=\Phi(t), \quad t \in[0, \infty], \\
u(x, y, t)_{(x, y) \rightarrow(0,0)} \longrightarrow \infty, \quad t \in[0, \infty] .
\end{gathered}
$$

The problem (6.5) for $g(t)=e^{t}$ with boundary and initially conditions is not invariant. But it is invariant for $g(t)=1$ and $g(t)=t^{b}, b>0$. As we found before the equation (1.1) with $g(t)=t^{b}$ has an infinitesimal generator

$$
X=\left(c_{1}+c_{2} x\right) \frac{\partial}{\partial x}+\left(c_{3}+c_{2} y\right) \frac{\partial}{\partial y}+c_{2} \frac{t}{b} \frac{\partial}{\partial t}+c_{2} \frac{b-\alpha}{b(n-1)} u \frac{\partial}{\partial u} .
$$

So, after applying $X$ to boundary condition as $\xi_{1}(0)=0$ for $x=0$ and $\xi_{2}(0)=0$ for $y=0$ we get $c_{1}=c_{3}=0$ and $c_{2}\left(\frac{b-1}{b(n-1)} \Phi(t)-t \frac{d \Phi}{d t}\right)=0$, where $\Phi(t)=K t^{\frac{b-1}{n-1}}, K$ is arbitrary constant.

According above definition let assume $t^{*}=t, x^{*}=1 / x, y^{*}=1 / y$, and $u^{*}=u$ bijective transformation which maps $M=\{x \rightarrow \infty, y \rightarrow \infty, u \rightarrow$ $\infty\}$ to $M^{*}=\left\{x^{*} \rightarrow 0, y^{*} \rightarrow 0, u^{*} \rightarrow 0\right\}$. This transformation maps the infinitesimal operator $X$ to $X^{*}$. Thus, $X=x \frac{\partial}{\partial x}+y \frac{\partial}{\partial y}+\frac{t}{b} \frac{\partial}{\partial t}+\frac{b-1}{b(n-1)} \frac{\partial}{\partial u}$ infinitesimal operator with $X^{*}=-x^{*} \frac{\partial}{\partial x^{*}}-y^{*} \frac{\partial}{\partial y^{*}}+\frac{t^{*}}{b} \frac{\partial}{\partial t^{*}}-\frac{b-1}{b(n-1)} u^{*} \frac{\partial}{\partial u^{*}}$ leaves invariant the equation (6.5) with boundary and initially conditions:

$$
\begin{aligned}
& u(t, x, y)_{t \rightarrow 0} \longrightarrow \infty, \quad(x, y) \in \mathbb{R}^{+} \times \mathbb{R}^{+}, \\
& u(t, 0,0)=K t^{\frac{b-1}{n-1}}, \quad t \in[0, \infty], \\
& u(t, x, y)_{(x, y) \rightarrow(0,0)} \longrightarrow \infty, \quad t \in[0, \infty] .
\end{aligned}
$$

Which give us $u=t^{\frac{b-1}{n-1}} f\left(r_{1}, r_{2}\right)$, where $r_{1}=\frac{x}{t^{b}}, r_{2}=\frac{y}{t^{b}}$ transformation, after applying that we get

$$
\left\{\begin{array}{l}
\frac{b-1}{n-1} f-b\left(r_{1} f_{r_{1}}+r_{2} f_{r_{2}}\right)+n P f^{n-1}\left(f_{r_{1}}+f_{r_{2}}\right)-R\left(n f^{n-1}\left(f_{r_{1} r_{1}}+f_{r_{2} r_{2}}\right)+n(n-1) f^{n-2}\left(f_{r_{1}}^{2}+f_{r_{2}}^{2}\right)\right)=0, \\
f\left(r_{1}, r_{2}\right)\left(r_{1}, r_{2}\right) \rightarrow(0,0) \longrightarrow 0, \\
\quad r_{\left(r_{1}, r_{2}\right) \longrightarrow(\infty, \infty)} f\left(r_{1}, r_{2}\right)=K,
\end{array}\right.
$$

boundary value problem of partial differential equation with two independent variable.

And the equation (1.1) with $g(t)=1$ have an infinitesimal generator

$$
X=c_{1} \frac{\partial}{\partial x}+c_{2} \frac{\partial}{\partial y}+\left(c_{3} t+c_{4}\right) \frac{t}{b} \frac{\partial}{\partial t}+c_{4} \frac{1}{1-n} \frac{\partial}{\partial u} .
$$

Thus, as $\xi_{1}(0)=0$ for $x=0, \xi_{2}(0)=0$ for $y=0$, and $\tau(0)=0$ for $t=0$ we get

$$
c_{1}=0, \quad c_{2}=0, \quad c_{4}=0, \quad \text { and } \quad c_{3}\left(\frac{1}{1-n} \Phi(t)-t \frac{d \Phi}{d t}\right)=0 .
$$

So, $\Phi(t)=C t^{\frac{1}{1-n}}, C$ is arbitrary constant. According above, the $X=t \frac{\partial}{\partial t}+\frac{1}{1-n} \frac{\partial}{\partial u}$ with $X^{*}=t^{*} \frac{\partial}{\partial t^{*}}-\frac{1}{1-n} u^{*} \frac{\partial}{\partial u^{*}}$ infinitesimal operator leaves invariant the equation (6.5) with boundary and initially conditions:

$$
\begin{aligned}
& u(t, x, y)_{t \rightarrow 0} \longrightarrow \infty, \quad(x, y) \in \mathbb{R}^{+} \times \mathbb{R}^{+}, \\
& u(t, 0,0)=C t^{\frac{1}{1-n}}, \quad t \in[0, \infty], \\
& u(t, x, y)_{(x, y) \rightarrow(0,0)} \longrightarrow \infty, \quad t \in[0, \infty] .
\end{aligned}
$$

Which give us $u=t^{\frac{1}{1-n}} h(x, y)$ transformation, after applying that we get

$$
\left\{\begin{array}{l}
\frac{1}{1-n} h+n P h^{n-1}\left(h_{x}+h_{y}\right)-R\left(n h^{n-1}\left(h_{x x}+h_{y y}\right)+n(n-1) h^{n-2}\left(h_{x}^{2}+h_{y}^{2}\right)\right)=0, \\
h(x, y)_{(x, y) \rightarrow(0,0)}^{\longrightarrow} 0, \\
\lim _{(x, y) \longrightarrow(\infty, \infty)} h(x, y)=C,
\end{array}\right.
$$

boundary value problem of partial differential equation with two independent variable. 


\section{Conclusion}

In this work, we presented the application of Lie group analysis to study time-fractional nonlinear generalized Burgers' differential equations. So, we found some exact solutions of nonlinear generalized Burgers' differential equation with fractional derivative here we used the method of Lie groups method. Also, we obtained the conservation laws for corresponding cases of the function $g(t)$. After applying the Lie groups we got boundary value problems with reduced dimension for special cases of $g(t)$. Moreover we defined conditions which leave invariant the boundary value problem (1.1)-(1.2) for $g(t)=t^{\lambda}$ and $g(t)=1$ with $\alpha=1$. The symmetry method on fractional boundary value problem is our future research.

\section{Acknowledgment}

The authors would like to thank Istanbul Commerce University, 22-2018/34 and Turkish scholarships for supporting this work.

\section{References}

[1] C. S. Gardner, J. M. Greene, M. D. Kruskal, R. M. Miura, Method for solving the Korteweg-de Vries equation, Phys. Rev. Lett., 19 (1967), 1095-1097.

[2] R. Hirota, J. Satsuma, A variety of nonlinear network equations generated from the Bäcklund transformation for the Tota lattice, Suppl. Prog. Theor. Phys., 59 (1976), 64-100.

[3] G. W. Bluman, S. C. Anco, Symmetry and integration methods for differential equations, 154 Appl. Math. Sci., Springer-Verlag, New York, 2002.

[4] P. Olver, Applications of Lie Groups to Differential Equations, Springer Science, Germany, 2012.

[5] P. Clarkson, M. Kruskal, New similarity reductions of the Boussinesq equation, J. Math. Phys., 30(10) (1989), $2201-2213$.

[6] P. Clarkson, New similarity reductions for the modified Boussinesq equation, J. Phys. A: Gen., 22 (1989), $2355-2367$.

[7] R. K. Gazizov, A. A. Kasatkin, S. Y. Lukashchuk, Continuous transformation groups of fractional differential equations, Vestn. USATU, 9 (2007), C. M. Khalique, K. R. Adem, Exact solutions of the $(2+1)$-dimensional Zakharov-Kuznetsov modified equal width equation using Lie group analysis, C. M. Khalique, K. R. Adem, Exact solutions of th
Math. Comp. Modelling, 54 (2011), 184-189.

[9] S. S. Ray, Invariant analysis and conservation laws for the time fractional $(2+1)$-dimensional Zakharov-Kuznetsov modified equal width equation using Lie group analysis, Comput. Math. Appl., 76 (2018), 2110-2118

[10] N. Heymans, I. Podlubny, Physical interpretation of initial conditions for fractional differential equations with Riemann-Liouville fractional derivatives, Rheol. Acta, 45(5) (2006), 765-771.

[11] C. Li, D. Qian, Y. Q. Chen, On Riemann-Liouville and Caputo derivatives, Discrete Dyn. Nat. Soc., 15 (2011), Article ID 562494.

[12] P. Hydon, Symmetry Methods for Differential Equations: A Beginner's Guide, Cambridge University press., UK, 2000.

[13] G. Iskandarova, D. Kaya, Symmetry solution on fractional equation, J. Optim. Control: Theories Appl., 7(3) (2017) 255-259.

[14] D. Kaya, G. Iskandarova, Lie group analysis for a time-fractional nonlinear generalized KdV differential equation, Turk. J. Math., 43(3) (2019),

[15] N. M. Ivanova, C. Sophocleous, R. Tracin, Lie group analysis of two-dimensional variable-coefficient Burgers equation, Z. Angew. Math. Phys., 61(5) (2010), 793-809.

[16] M. Abd-el-Malek, A. Amin, Lie group method for solving the generalized Burgers', Burgers'-KdV and KdV equations with time-dependent Kiryakiable coefficients, J. Symmetry, 7 (2015), 1816-1830.

[17] A. Yokus, M. Yavuz, Novel comparison of numerical and analytical methods for fractional Burger-Fisher equation, Discrete Contin. Dyn. Syst., (2020), (in press).

[18] R. Sinuvasan, K. M. Tamizhmani, P. G. L. Leach, Algebraic resolution of the Burgers equation with a forcing term, Pramana - J. Phys. 88(5) (2017), 74 pages.

[19] I. Podlubny, Fractional Differential Equations: An Introduction to Fractional Derivatives, Fractional Differential Equations, Some Methods of Their Solution and Some of Their Applications, Academic Press, San Diego, 1999.

[20] N. Ibragimov, Lie group analysis classical heritage, ALGA Publications Blekinge Institute of Technology Karlskrona, Sweden, 2004.

[21] N. Ibragimov, CRC Handbook of Lie Group Analysis of Differential Equations, 1 CRC Press, Boca Raton, 1994.

[22] K. S. Miller, B. Ross, An Introduction to the Fractional Calculus and Fractional Differential Equations, Wiley-Interscience, New York, 1993.

[23] K. B. Oldham, J. Spanier, The Fractional Calculus, Academic Press, New York, 1974.

[24] N. Ibragimov, A new conservation theorem, J Math. Anal. Appl., 333(1) (2007), 311-328.

[25] N. Ibragimov, Nonlinear self-adjointness and conservation laws, J. Phys A: Math. Gen., 44(43) (2011), 4109-4112.

[26] Z. Xiao, L. Wei, Symmetry analysis conservation laws of a time fractional fifth-order Sawada-Kotera equation, J. Appl. Anal. Comput., 7 (2017), $1275-1284$

[27] S. Y. Lukashchuk, Conservation laws for time-fractional subdiffusion and diffusion-wave equations, Nonlinear Dyn., 80(1-2) (2015), 791-802.

[28] R. K. Gazizov, N. H. Ibragimov, S. Y. Lukashchuk, Conlinear self-adjointness, conservation laws and exat solution of fractional Kompaneets equations, Commun. Nonlinear SCI, 23(1) (2015), 153-163.

[29] G. W. Bluman, S. Kumei, Symmetries and Differential Equations, Berlin etc., Springer-Verlag, 1989.

[30] R. Cherniha, S. Kovalenko, Lie symmetry of a class of nonlinear boundary value problems with free boundaries, Banach Center Publ., 93 (2011), $73-82$.

[31] R. Cherniha, S. Kovalenko, Lie symmetries of nonlinear boundary value problems, Commun. Nonlinear SCI, 17 (2012), 71-84. 